\title{
Mössbauer spectroscopy investigation of Mn-substituted Co-ferrite $\left(\mathrm{CoMn}_{x} \mathrm{Fe}_{2-x} \mathrm{O}_{4}\right)$
}

\author{
K. Krieble ${ }^{\text {a) }}$ and T. Schaeffer \\ Moravian College, Bethlehem, Pennsylvania 18018 \\ J. A. Paulsen, A. P. Ring, and C. C. H. Lo \\ Center for Nondestructive Evaluation, Iowa State University, Ames, Iowa 50011 \\ J. E. Snyder \\ Materials and Engineering Physics Program, Ames Laboratory, U.S. Department of Energy, and Materials \\ Science and Engineering Department, Iowa State University, Ames, Iowa 50011
}

(Presented on 8 November 2004; published online 10 May 2005)

\begin{abstract}
Understanding the effect of Mn substitution for Fe in Co ferrite presents a challenge because there are three different transition-metal ions distributed among two distinct crystallographic and magnetic sublattices with complicated superexchange and anisotropic interactions. In this study, a series of six powder samples with compositions $\mathrm{Co}_{1.0} \mathrm{Mn}_{x} \mathrm{Fe}_{2-x} \mathrm{O}_{4}$ were investigated using transmission Mössbauer spectroscopy. Mössbauer spectroscopy provides an excellent tool for probing the local environment of the $\mathrm{Fe}$ atoms present in such materials. Results show two sets of six-line hyperfine patterns for all samples, indicating the presence of $\mathrm{Fe}$ in both $A$ and $B$ sites. Identification of sites is accomplished by evidence from hyperfine distribution width, integrated intensity, and isomer-shift data. Increasing Mn concentration was found to decrease the hyperfine field strength at both sites, but at unequal rates, and to increase the distribution width. This effect is due to the relative strengths of $\mathrm{Fe}-\mathrm{O}-X$ superexchange $(X=\mathrm{Fe}, \mathrm{Co}$, or $\mathrm{Mn})$ and the different numbers of the next-nearest neighbors of $A$ and $B$ sites. Results are consistent with a model of $\mathrm{Mn}$ substituting into $B$ sites and displacing Co ions onto $A$ sites. (C) 2005 American Institute of Physics. [DOI: $10.1063 / 1.1846271]$
\end{abstract}

\section{INTRODUCTION}

Spinel ferrite magnetic oxides have received considerable research and applications attention for soft magnetic material and high-frequency applications; however, cobaltcontaining spinels have received relatively little attention due to their high magnetomechanical coupling and magnetostriction. Recently, however, it has been recognized that it is just those properties that make cobalt ferrite, substituted cobalt ferrite, and their metal-bonded composites as promising materials for magnetomechanical strain sensing and actuating applications. ${ }^{1}$ Substitution of other elements, such as manganese into cobalt ferrites, has been proposed in order to tailor the magnetic and magnetomechanical properties. ${ }^{2}$ Previous investigations of manganese-doped cobalt ferrites have concentrated on the study of thin films and fine particles for magneto-optical applications. ${ }^{3,4}$ In the present study, we report on the magnetic characterization of manganese-doped cobalt ferrite powders as a function of manganese concentration using transmission Mössbauer spectroscopy.

\section{EXPERIMENTAL DETAILS}

A series of manganese-doped cobalt ferrite powder samples with compositions $\mathrm{CoFe}_{2-x} \mathrm{Mn}_{x} \mathrm{O}_{4}$ (where $x$ ranges from 0.0 to 0.8 ) were prepared by substituting manganese for iron. The samples were made using standard powder ceramic techniques, mixing $\mathrm{Fe}_{2} \mathrm{O}_{3}, \mathrm{MnO}_{2}$, and $\mathrm{Co}_{3} \mathrm{O}_{4}$ powders in the

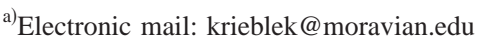

proper ratios for the desired cation concentrations. The powder was mixed, calcined, ball milled, mixed, and calcined again. The powder was then remilled, mixed, and sintered in air. The samples were then cooled by removal from the furnace to room temperature, then reground to form the powders used in this study. Samples were mounted for transmission Mössbauer measurements by encasing a $0.5-\mathrm{mm}$-thick layer of powder between two sheets of plastic tape.

The Mössbauer spectroscopy system used in this study was operated in transmission geometry mode. The major components of the Mössbauer system include an Austin Science Associates (ASA) model S3 Mössbauer spectrometer drive, an ASA K3 Linear Motor drive unit, an ASA CSP 200 gas proportional detector, a Canberra PCA-3 multichannel analyzer computer board which is synchronized with the drive unit, and a $15 \mathrm{mCi} \mathrm{Co}^{57}(\mathrm{Rh})$ source.

\section{DISCUSSION}

In Fig. 1 Mössbauer spectra of the six samples are shown with manganese concentration increasing from bottom to top $(X=0.0,0.2,0.3,0.4,0.6$, and 0.8$)$. The spectra show the presence of two distinct six line hyperfine patterns, indicating two different types of ferromagnetic $\mathrm{Fe}$ atoms in the structure. These can be identified as Fe in the $A$ (tetrahedral) and $B$ (octahedral) site locations within the spinel structure. As the manganese concentration increases, the line splitting (indicative of the magnetic hyperfine field at the $\mathrm{Fe}$ nuclei, and related to the exchange coupling) can be seen to 


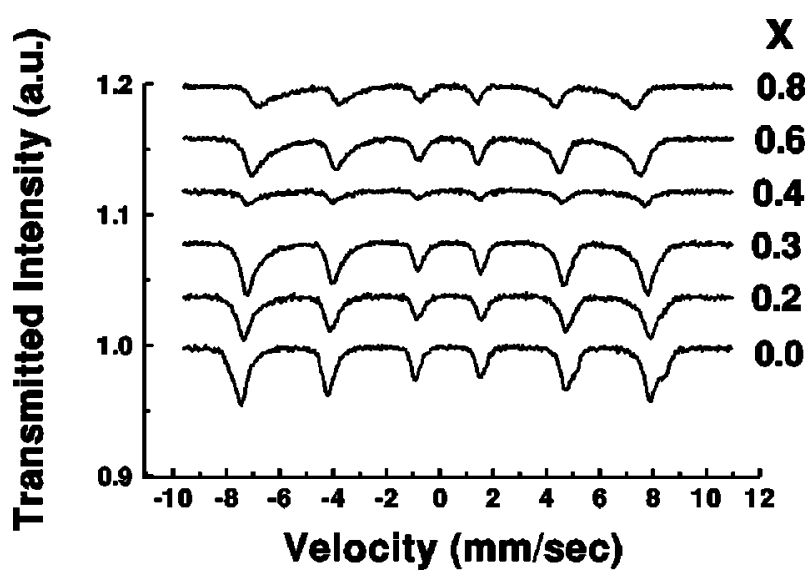

FIG. 1. Raw transmission Mössbauer data for $\mathrm{CoFe}_{2-x} \mathrm{Mn}_{x} \mathrm{O}_{4}$ where (bottom to top) $x=0.0,0.2,0.3,0.4,0.6$, and 0.8 .

decrease. In addition, the individual six line patterns vary relative to one another, indicating a change in the relative magnetic environments of $\mathrm{Fe}$ in the $A$ and $B$ sites.

To extract quantitative values for the hyperfine field strength, distribution width, and isomer shift, a curve-fitting process using WMOSS software was applied to the raw data. A composite of two hyperfine patterns was used because the spectra appear to be composed of two sets of lines, corresponding to iron in the $A$ or $B$ site locations. Figure 2 shows the results of one such fit for the $\mathrm{CoFe}_{1.4} \mathrm{Mn}_{0.6} \mathrm{O}_{4}$ sample. The two individual hyperfine patterns are shown above the data, and the best-fit curve (which is their sum) is shown overlapping the raw data points (+'s).

The software uses a standard six line spectrum with peaks constructed using a Lorentzian profile. The hyperfine field is used as a variable parameter, as is the isomer shift, which measures the overall shift of the spectrum from the zero velocity location. A Gaussian distribution of hyperfine fields is assumed for each pattern, with which the basic Lorentzian line shape is convolved. This Gaussian distribution represents the fact that the iron atoms in the same crystallographic site will not have an identical set of next-nearest neighbors to which they are magnetically coupled by superexchange, but will have a statistical distribution of neighbors and therefore hyperfine field strengths. The calculated value

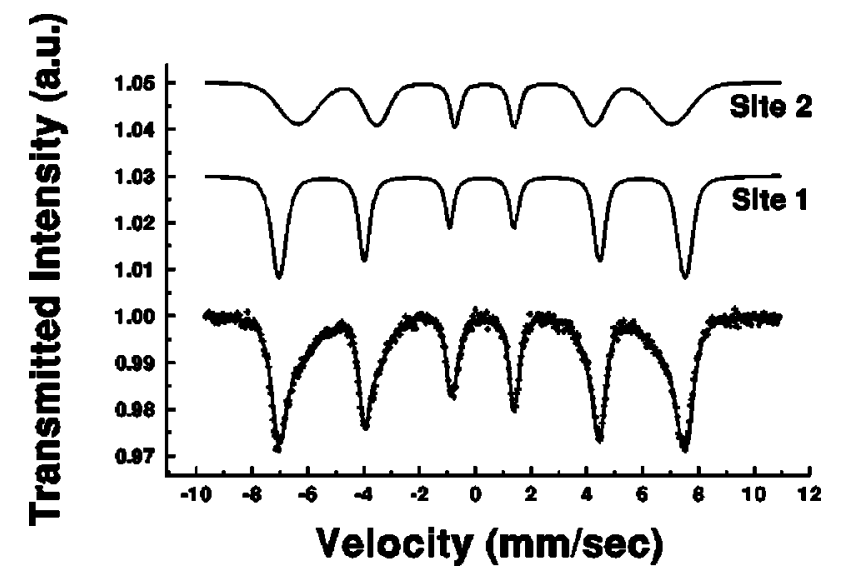

FIG. 2. Experimental Mössbauer data and fit with two six-line hyperfine patterns for $\mathrm{CoFe}_{1.4} \mathrm{Mn}_{0.6} \mathrm{O}_{4}(x=0.6)$.

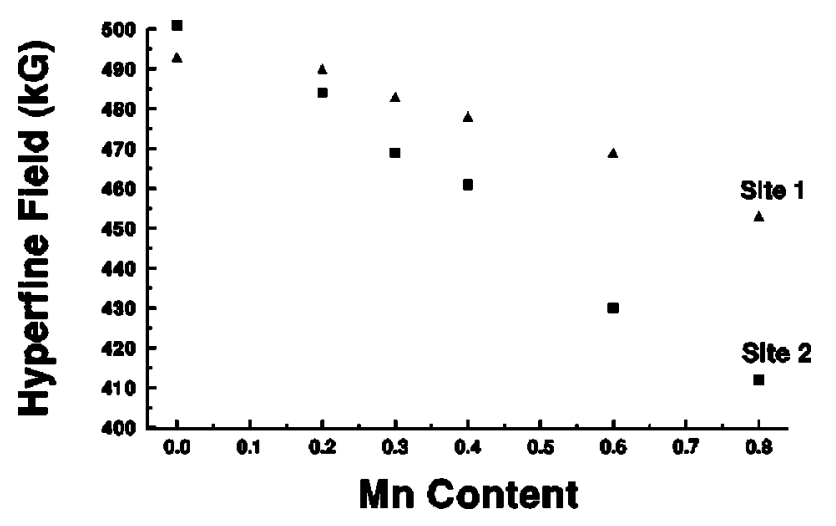

FIG. 3. Hyperfine field strength as a function of $\mathrm{Mn}$ content $(x)$ in $\mathrm{CoFe}_{2-x} \mathrm{Mn}_{x} \mathrm{O}_{4}$ (see text for discussion of identification of $A$ and $B$ sites).

for the hyperfine field represents the centroid of the Gaussian distribution. Results of the fits are shown in Figs. 3-5.

An important step in the analysis is the identification of which pattern corresponds to the $A$ sites, and which to the $B$. Considering first the pure cobalt ferrite $(x-0.0)$, hyperfine field by itself is not a reliable way to determine this, since in some studies $A$ is reported to have the higher hyperfine field, ${ }^{5}$ and in some $B .^{6}$ Following the model of Sawatzky et $a l .{ }^{7,8}$ this apparent discrepancy can be understood, if one assumes different distributions of next-nearest neighbors. The antiferromagnetic $A-B$ coupling is generally thought to be the strongest coupling in the structure. Each $A$-site Fe is coupled by superexchange to $12 B$ next-nearest neighbors, but each $B$-site Fe is coupled to only $6 A$ nearest neighbors. Therefore the $B$ site hyperfine distribution width and position of the centroid are much more affected by where the Co goes than is the $A$ distribution, and the $B$-site width for the pure Co ferrite should be greater than the $A$-site width. ${ }^{7}$ Thus the pattern with the wider distribution at $x=0.0$ (site 2) can be identified as that corresponding to the $B$ sites (see Fig. 4). The ratio of integrated areas under the two patterns for the pure Co ferrite is consistent with this identification. In addition, the isomer-shift results also support this identification (Fig. 5). The hyperfine pattern identified as $A$ sites (site 1) shows an isomer shift of $0.14 \mathrm{~mm} / \mathrm{s}$ for $x=0.0$ and a range of $0.14-0.23 \mathrm{~mm} / \mathrm{s}$ with added $\mathrm{Mn}$. The pattern identified as $B$ sites (site 2) shows an isomer shift of $0.30 \mathrm{~mm} / \mathrm{s}$ for $x$

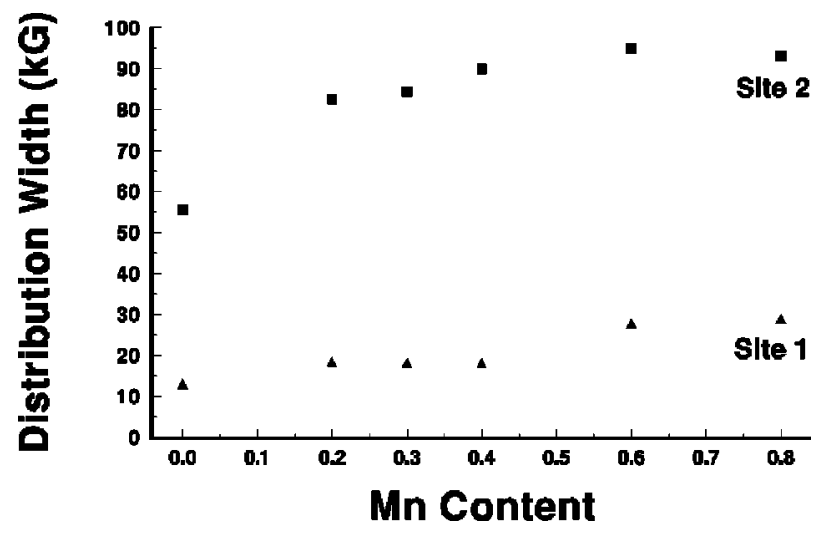

FIG. 4. Hyperfine distribution width as a function of Mn content $(x)$ in $\mathrm{CoFe}_{2-x} \mathrm{Mn}_{x} \mathrm{O}_{4}$ (see text for discussion of identification of $A$ and $B$ sites). 


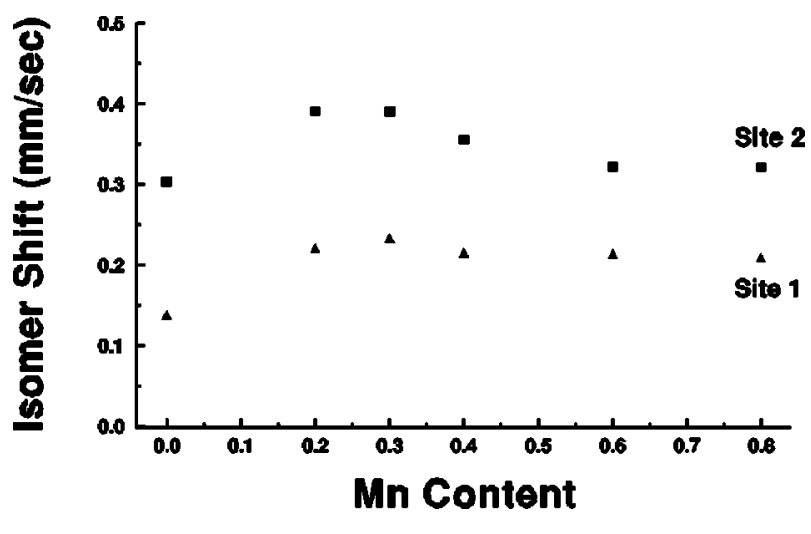

FIG. 5. Isomer shift as a function of Mn content $(x)$ in $\mathrm{CoFe}_{2-x} \mathrm{Mn}_{x} \mathrm{O}_{4}$.

$=0.0$ and a range of $0.30-0.39 \mathrm{~mm} / \mathrm{s}$ with added $\mathrm{Mn}$. The expected range of $\mathrm{Fe}^{3+}$ with oxygen coordination is given in Ref. 9 to be $0.20-0.30 \mathrm{~mm} / \mathrm{s}$, with an average of about $0.22 \mathrm{~mm} / \mathrm{s}$ for tetrahedral oxygen coordination; and $0.30-0.46 \mathrm{~mm} / \mathrm{s}$, with an average of about $0.35 \mathrm{~mm} / \mathrm{s}$ for octahedral coordination. Thus the isomer-shift data also agree with the identification of $A$ and $B$ sites.

Having now identified the $A$-site and $B$-site patterns, consider the effect of Mn substitution for Fe in Figs. 3 and 4. Substituting $\mathrm{Mn}$ for $\mathrm{Fe}$ has the overall effect of decreasing the hyperfine field at both the $A$ sites and $B$ sites. This can be understood assuming that the $\mathrm{Mn}-\mathrm{O}-\mathrm{Fe}$ superexchange is not as strong as the $\mathrm{Fe}-\mathrm{O}-\mathrm{Fe}$ superexchange. The hyperfine field identified with $B$ sites decreases at a steeper rate than $A$, and the width of its distribution increases faster. In substituting $\mathrm{Mn}$ for $\mathrm{Fe}$ in $\mathrm{CoFe}_{2} \mathrm{O}_{4}$, we assume that $\mathrm{Mn}^{+3}$ is substituting for $\mathrm{Fe}^{+3}$. Of the three cations $\mathrm{Co}^{+2}, \mathrm{Fe}^{+3}$, and $\mathrm{Mn}^{+3}$, $\mathrm{Mn}^{+3}$ is reported to have the strongest octahedral preference, and $\mathrm{Fe}^{3+}$ to have the strongest tetrahedral preference (with $\mathrm{Co}^{+2}$ intermediate). ${ }^{10}$ (Note that our isomer-shift values indicate that the $\mathrm{Fe}$ is indeed in the $\mathrm{Fe}^{+3}$ oxidation state, as $\mathrm{Fe}^{+2}$ with oxygen coordination has much higher isomer-shift values. ') It appears that Mn substitution is not merely replacing $\mathrm{Fe}$ in the $B$ sites (which might be expected to affect the $A$-site hyperfine field and distribution width more than $B$, since $A-B$ coupling is the strongest), but rather that it has the effect of displacing Co ions to the $A$ sites (and according to Ref. 7, Fe-O-Co superexchange appears weaker than $\mathrm{Fe}-$ $\mathrm{O}-\mathrm{Fe}$ superexchange). Indeed, that is what is shown in the cation distributions proposed for the slow-cooled samples of Ref. 6. And increasing the amount of Co on $A$ sites has been shown to increase the width and decrease the centroid of the hyperfine field distribution in pure Co ferrite. ${ }^{7} B$-site hyperfine field and width are much more easily affected than $A$ by exchanging $\mathrm{Fe}-\mathrm{O}-\mathrm{Fe}$ superexchange links for $\mathrm{Fe}-\mathrm{O}-X$, since each $B$ is only linked to $6 A$ next-nearest neighbors, whereas each $A$ is linked to $12 B \mathrm{ss}^{7}$ The $A$-site width and hyperfine field are also affected by the substitution, but to a lesser extent. The relative rates of change of the hyperfine field and width of $A$ and $B$ sites as a function of Mn substi- tution will be influenced by both the different numbers of next-nearest neighbors linked by superexchange and the statistical distribution of the three different cations populating those sites, and by the relative strengths of $\mathrm{Fe}^{+3}-\mathrm{O}-\mathrm{Fe}^{+3}$, $\mathrm{Fe}^{+3}-\mathrm{O}-\mathrm{Co}^{+2}$, and $\mathrm{Fe}^{+3}-\mathrm{O}-\mathrm{Mn}^{+3}$ superexchange.

\section{CONCLUSIONS}

The effects of manganese concentration on the magnetic hyperfine characteristics of Mn-substituted Co ferrites have been studied using Mössbauer spectroscopy. Separate contributions due to the $A$ (tetrahedral) and $B$ (octahedral) sites can be observed. They can be identified by hyperfine field distribution width, isomer shift, and integrated area under the hyperfine field pattern. The results indicate the reduction of magnetic hyperfine field strength and increase of magnetic hyperfine field distribution as manganese is substituted for iron. The $B$-site hyperfine field and distribution are affected more than the $A$ site. This is consistent with a hypothesis that the Mn substitutes into $B$ sites and displaces Co onto $A$ sites. Further measurements and more detailed analysis should help elucidate how changes in Mn content affect the magnetic and magnetomechanical properties of these materials, providing the knowledge base for future applications of the materials.

\section{ACKNOWLEDGMENTS}

This work was supported in part by the by the National Science Foundation, Division of Materials Research under Grant No. DMR-0402716 and in part by the National Aeronautical and Space Administration (NASA) under Award No. NAG-1-02098. Samples were fabricated in collaboration with the Ames Laboratory Materials Preparation Center, which is supported by the U.S. Department of Energy, Office of Basic Energy Sciences. Ames Laboratory is operated by the U.S. Department of Energy by Iowa State University under Contract No. W-7405-ENG-82.

${ }^{1}$ Y. Chen, J. E. Snyder, C. R. Schwichtenberg, K. W. Dennis, R. W. McCallum, and D. C. Jiles, IEEE Trans. Magn. 35, 3652 (1999).

${ }^{2}$ J. A. Paulsen, A. P. Ring, C. C. H. Lo, J. E. Snyder, and D. C. Jiles, Presentation W27-5, American Physical Society March Meeting, Montreal, Quebec, Canada, March 2004 (unpublished).

${ }^{3}$ B. Zhou, Y.-W. Zhang, C.-S. Liao, F.-X. Cheng, C.-H. Yan, L.-Y. Chen, and S.-Y. Wang, Appl. Phys. Lett. 79, 1849 (2001).

${ }^{4}$ B. Zhou, Y.-W. Zhang, C.-S. Liao, and C.-H. Yan, J. Magn. Magn. Mater. 247, 70 (2002).

${ }^{5}$ D. H. Lee, H. S. Kim, C. H. Yo, K. Ahn, and K. H. Kim, Mater. Chem. Phys. 57, 169 (1998).

${ }^{6}$ M. K. Fayek, F. M. Sayed Ahmed, S. S. Ata-Allah, M. K. Elnimer, and M. F. Mostafa, J. Mater. Sci. 27, 4813 (1992).

${ }^{7}$ G. A. Sawatzky, F. van der Woude, and A. H. Morrish, J. Appl. Phys. 39, 1204 (1968).

${ }^{8}$ G. A. Sawatzky, F. van der Woude, and A. H. Morrish, Phys. Rev. 187, 747 (1969).

${ }^{9}$ A. G. Maddock, Mössbauer Spectroscopy Principles and Applications of the Techniques (Horwood Publishing Limited, Chichester, 1997).

${ }^{10}$ R. C. O'Handley, Modern Magnetic Materials Principles and Applications (Wiley, New York, 2000). 